distinguish the early initiators of NEC from the later consequences of the disease pathology. To elucidate the mechanisms and identify clinical interventions, animal models showing spontaneous NEC development may help. In this review, we summarize some recent results from studies on preterm pigs during the early feedinginduced mucosal dysfunction and later NEC development. We show that introduction of suboptimal enteral formula diets, coupled with parenteral nutrition, predispose to disease, while advancing amounts of mother's milk from birth protects against disease. Hence, the transition from parenteral to enteral nutrition shortly after birth plays a pivotal role to secure gut growth, digestive maturation and an appropriate response to bacterial colonization in the sensitive gut of preterm neonates. Ongoing studies in preterm pigs aim to identify the optimal time, amount and diet of the first enteral milk that best secure both early gut adaptation and later body growth and health.

\section{PREVENTION OF NECROTISING ENTEROCOLITIS}

doi:10.1136/archdischild-2012-302724.0183

1.2S Patole. 'Neonatal Paediatrics, KEM Hospital for Women; ${ }^{2}$ Centre for Neonatal Research and Education, University of Western Australia, Perth, WA, Australia

Necrotising enterocolitis (NEC) is a potentially disastrous illness that occurs in $6-8 \%$ of preterm (gestation $<32$ weeks) very low birth weight neonates. The mortality $(\sim 25 \%)$ and morbidity of $\geq$ Stage II NEC (e.g. need for surgery, survival with short bowel syndrome with protracted feed intolerance, complications of prolonged dependence on parenteral nutrition, recurrent infections, and prolonged hospital stay) is significant. The incidence (10-12\%), mortality (40-45\%), and morbidity including need for surgery, and risk of long-term neurodevelopmental impairment after surviving surgery for NEC is worse in extremely low birth weight neonates. The economic burden of NEC is substantial ( $~ 500$ million to 1 billion dollars per year in the USA) considering the prolonged hospital stay due to the associated complications. NEC has become one of the common causes of death in preterm neonates surviving the first week of life. The pathogenesis of NEC remains poorly understood despite decades of research. Prevention of prematurity, the single most important risk factor for the illness, has proved to be a difficult task. The absolute number of preterm neonates at risk for the illness has increased with advances in neonatal intensive care. Prevention of NEC has thus become a priority. The well established (e.g. antenatal glucocorticoids, early preferential feeding with breast milk, standardised feeding protocols) as well as newer strategies (e.g. probiotics, prebiotics) for primary as well as potentially secondary (e.g. pentoxifylline, bosentan) prevention of NEC will be reviewed.

\section{INTRAPERITONEAL CYTIDINE 5'-DIPHOSPHOCHOLINE (CDP-CHOLINE) ADMINISTRATION REDUCES THE SEVERITY OF INTESTINAL INJURY IN A NEONATAL RAT MODEL OF NECROTIZING ENTEROCOLITIS}

doi:10.1136/archdischild-2012-302724.0184

'M Cetinkaya, ${ }^{2} \mathrm{M}$ Cansev, ' $1 \mathrm{~F}$ Cekmez, ' $\mathrm{C}$ Tayman, ' $\mathrm{FE}$ Canpolat, ${ }^{2} \mathrm{M}$ Kafa, ${ }^{3} \mathrm{~S}$ Uysal, ${ }^{1} \mathrm{SU}$ Sarıı. ' GATA Teaching Hospital, Ankara; ${ }^{2}$ Uludağ University Medical Faculty, Bursa; ${ }^{3}$ Fatih University Medical Faculty, Ankara, Turkey

Background The aim of this study was to evaluate the possible cytoprotective effect of CDP-choline treatment on intestinal cell death and apoptosis in a neonatal rat model of NEC.

Methods A total of 30 newborn pups were divided equally into 3 groups as follows: Control, NEC, and NEC+CDP-choline groups. NEC was induced by enteral formula feeding, exposure to hypoxiahyperoxia and cold stress. CDP-choline was administered intraperitoneally at a dose of $300 \mathrm{mg} / \mathrm{kg} /$ day for 3 days starting from the first day of life. Macroscopical, histopathological, ibflammatory markers, caspase- 3 expression and apoptosis were evaluated on the gut samples. Activities of xanthine oxidase, superoxide dismutase, glutathione peroxidase, malondialdehyde and myeloperoxidase were determined.

Results Median clinical sickness score, macroscopic gut assessment score and intestinal injury score were significantly improved in pups in NEC+CDP-choline group. In contrast, median apoptosis score was significantly higher in NEC group compared with NEC+CDP-choline group. Proinflammatory cytokine concentrations (IL-1 $\beta$, IL- 6 and TNF- $\alpha$ ) and caspase-3 expression in the intestinal tissue of the NEC+CDP-choline group were significantly lower. Moreover, tissue GSH-Px and SOD activities were preserved, whereas tissue MDA content, $\mathrm{MPO}$ and $\mathrm{XO}$ activities were significantly lower in NEC+CDP-choline group.

Conclusion This is the first study to report beneficial effects of CDP-choline treatment on intestinal injury in a neonatal rat model of NEC. Intraperitoneal CDP-choline administration significantly reduced clinical sickness score, ameliorated macroscopic and histopathological intestinal injury, reduced the inflammation and decreased apoptosis. These data suggest that, CDP-choline may be used as an effective therapeutic agent for prevention of NEC.

\section{MESENTERIC ARTERY REACTIVITY AND SMALL INTESTINE MORPHOLOGY IN A CHICKEN MODEL OF HYPOXIA- INDUCED FETAL GROWTH RESTRICTION}

doi:10.1136/archdischild-2012-302724.0185

${ }^{1}$ RM Moonen, ${ }^{2}$ CG Kessels, ${ }^{2}$ E Villamor. 'Pediatrics, Atrium Medical Center Parkstad, Heerlen; ${ }^{2}$ Pediatrics, Maastricht University Medical Center, Maastricht, The Netherlands

Background and aims Infants with intrauterine growth retardation are prone to intestinal dysfunction which is manifested by feeding intolerance and, in the most severe cases, necrotizing enterocolitis. The morphological and molecular mechanisms that lead to these complications are not completely understood and suitable experimental models are necessary. We aimed to characterize mesenteric artery $(\mathrm{MA})$ reactivity, small intestine morphometry and intestinal expression of VEGF in a chicken model of hypoxiainduced fetal growth restriction.

Methods Chicken embryos (15 and 19 days) and hatchlings $\left(<3\right.$-h-old and 1-d-old) were incubated under hypoxic $\left(15 \% \mathrm{O}_{2}\right.$ from day 0 to day 19 of incubation) or normoxic conditions. Vascular reactivity was studied using wire miography. Intestinal morphometry was assessed in hematoxyline-eosine-stained sections. The expression of VEGF mRNA was determined by RTPCR analysis.

Results Hypoxia altered the responsiveness of chicken embryo MAs to acetylcholine, the NO donor sodium nitroprusside and the constrictor polypeptide ET-1. However, the majority of these alterations, with the exception of the hyperresponsiveness to ET-1, were not present in the hypoxic hatchlings. When intestinal histology was analyzed, subtle hypoxia-induced changes were noted in the muscularis propia and the villi from the hatchlings. Hypoxic incubation also diminished the expression of VEGF mRNA in the terminal ileum of the hatchlings.

Conclusions Chronic moderate hypoxia during incubation results in subtle but significant alterations in chicken MA reactivity, small intestine morphology and VEGF expression. Whether these alterations may have a direct effect on the functional status of the intestine remains to be investigated.

\section{PROTECTIVE EFFECTS OF COLCHICINE IN AN EXPERIMENTAL MODEL OF NECROTIZING ENTEROCOLITIS IN NEONATAL RAT}

doi:10.1136/archdischild-2012-302724.0186 